Revue d'histoire de l'Amérique française

REVUE D.HISTOIRE DE L'AMÉRIQUE FRANÇAISE

\title{
Entre discours et pratiques les médecins montréalais face à la thérapeutique, 1869-1890
}

\section{Johanne Collin}

Volume 53, numéro 1, été 1999

Médecine, santé et sociétés

URI : https://id.erudit.org/iderudit/005360ar

DOI : https://doi.org/10.7202/005360ar

Aller au sommaire du numéro

\section{Éditeur(s)}

Institut d'histoire de l'Amérique française

ISSN

0035-2357 (imprimé)

1492-1383 (numérique)

Découvrir la revue

Citer cet article

Collin, J. (1999). Entre discours et pratiques les médecins montréalais face à la thérapeutique, 1869-1890. Revue d'histoire de l'Amérique française, 53(1), 61-92. https://doi.org/10.7202/005360ar
Résumé de l'article

Dans la perspective large de questionner les aspects sociaux et culturels du recours aux médicaments à différentes époques, cet article se penche sur l'usage de la thérapeutique dans la seconde moitié du XIXe siècle. Il vise à mieux cerner la place du médicament dans les pratiques de santé à une époque charnière du développement de la pharmacothérapie et notamment à scruter les pratiques médicales à cet égard. Qu'en est-il des attitudes des médecins québécois, et plus spécifiquement des médecins montréalais, face à la thérapeutique au cours des dernières décennies du XIXe siècle? Comment prescrivent-ils? Que prescrivent-ils? Comment se situe la réalité de leurs pratiques par rapport au discours médical qui a jusqu'ici constitué la principale source pour saisir l'utilisation de la thérapeutique au XIXe siècle? Ce sont les questions auxquelles nous cherchons réponse à travers l'analyse croisée de deux sources : l'ordonnancier d'un pharmacien montréalais (Henry R. Gray) qui témoigne des pratiques réelles et quotidiennes de prescription des médecins et la presse médicale montréalaise qui permet de retracer le discours médical sur l'usage de la thérapeutique entre 1869 et 1890.
Tous droits réservés @ Institut d'histoire de l'Amérique française, 1999
Ce document est protégé par la loi sur le droit d'auteur. L'utilisation des services d'Érudit (y compris la reproduction) est assujettie à sa politique d'utilisation que vous pouvez consulter en ligne.

https://apropos.erudit.org/fr/usagers/politique-dutilisation/ 


\title{
ENTRE DISCOURS ET PRATIQUES LES MÉDECINS MONTRÉALAIS FACE À LA THÉRAPEUTIQUE, 1869-1890
}

\author{
JOHANNE COLLIN \\ Faculté de pharmacie \\ Secteur de socio-économie du médicament \\ Groupe de recherche sur les aspects sociaux \\ de la santé et de la prévention (GRASP) \\ Université de Montréal
}

\section{RÉSUMÉ}

Dans la perspective large de questionner les aspects sociaux et culturels du recours aux médicaments à différentes époques, cet article se penche sur l'usage de la thérapeutique dans la seconde moitié du XIX ${ }^{\mathrm{e}}$ siècle. Il vise à mieux cerner la place du médicament dans les pratiques de santé à une époque charnière du développement de la pharmacothérapie et notamment à scruter les pratiques médicales à cet égard. Qu'en est-il des attitudes des médecins québécois, et plus spécifiquement des médecins montréalais, face à la thérapeutique au cours des dernières décennies du XIX ${ }^{\mathrm{e}}$ siècle? Comment prescrivent-ils? Que prescrivent-ils? Comment se situe la réalité de leurs pratiques par rapport au discours médical qui a jusqu'ici constitué la principale source pour saisir l'utilisation de la thérapeutique au XIX $\mathrm{X}^{\mathrm{e}}$ siècle? Ce sont les questions auxquelles nous cherchons réponse à travers l'analyse croisée de deux sources : l'ordonnancier d'un pharmacien montréalais (Henry R. Gray) qui témoigne des pratiques réelles et quotidiennes de prescription des médecins et la presse médicale montréalaise qui permet de retracer le discours médical sur l'usage de la thérapeutique entre 1869 et 1890.

\section{ABSTRACT}

This paper focuses on medication use in the second half of the nineteenth century and is based on the parallel analysis of two main sources: the prescription books (50 volumes) of a Montreal druggist (Henry R. Gray) and the medical and scientific journals published in Montreal between 1869 and 1890. It aims at tracing the evolution of pharmacopoeia and the composition of drugs prescribed during this important period of expansion of pharmacology. It also aims at comparing the discourse and the practices of physicians, to capture better their attitudes toward drug therapy in the context of scientific innovation that characterizes the final decades of the nineteenth century, and to determine the extent and pace of its integration into everyday medicine. 
Cet article porte sur l'usage de la thérapeutique dans la seconde moitié $\mathrm{du} \mathrm{XIX}^{\mathrm{e}}$ siècle. Il vise à mieux cerner la place du médicament dans les pratiques de santé à une époque charnière du développement de la pharmacothérapie et, notamment, à scruter les pratiques médicales à cet égard. Dans l'histoire de la thérapeutique, le XIX ${ }^{\mathrm{e}}$ siècle est en effet considéré comme une période singulièrement marquée par le passage d'un « hyperactivisme » dans le recours aux médicaments à un scepticisme, voire à un nihilisme thérapeutique. À des moments différents, selon les sociétés, les médecins auraient ainsi été amenés à bannir de leurs pratiques l'utilisation abondante et intempestive de substances et de traitements violents tels que saignées et purgations ${ }^{1}$. Devant les échecs répétés de la «médecine héroïque » et les constats d'inefficacité soutenus par une statistique médicale qui s'organise, le corps médical aurait versé, à des degrés divers, dans la pratique d'une médecine expectante, c'est-à-dire d'une médecine tout entière axée sur la prévention et fondée sur le pouvoir de guérir de la nature. Selon cette position, le recours au médicament pour soigner serait minimal et n'interviendrait que pour soutenir la nature dans son œuvre de guérison; pour aider en somme le corps à guérir de lui-même et à recouvrer son équilibre. Le primat de la natura medicatrix aurait entraîné le rejet d'un arsenal thérapeutique désormais considéré comme inutile et dangereux ${ }^{2}$.

Les dernières décennies du XIX ${ }^{\mathrm{e}}$ siècle sont pourtant le théâtre d'un renouveau à cet égard. Les progrès de la chimie et de l'étude des effets physiologiques des substances médicamenteuses préfigurent l'entrée dans la modernité thérapeutique que caractérisent l'antibiothérapie, la multiplication des vaccins et le déploiement d'un arsenal capable d'éradiquer des maladies infectieuses contagieuses et mortelles.

Ainsi, sous l'impulsion du physiologiste français François Magendie (1783-1855), il y a avancement notable dans la détermination de l'action spécifique de drogues pures. Considérant que l'utilisation de préparations galéniques et de mixtures impures provenant d'extraits de plantes ne pou-

1. Voir Erwin H. Ackerknecht, Therapeutics from the Primitives to the Twentieth Century (New York, Harper, 1973). Également du même auteur : « Aspects of the History of Therapeutics », Bulletin of the History of Medicine, 36 (1962) : 389-419; Alex Berman, « The Heroïc Approach in $19^{\text {th }}$ Century Therapeutics », dans Judith W. Leavitt et Ronald L. Numbers, dir., Sickness and Health in America : Readings in the History of Medicine and Public Health (Madison, University of Wisconsin Press, 1978); Charles E. Rosenberg, « The Therapeutic Revolution : Medicine, Meaning and Social Change », dans Morris J. Vogel et C. H. Rosenberg, dir., The Therapeutic Revolution : Essays in the Social History of American Medicine (Philadelphia, University of Pensylvania Press, 1979); John H. Warner, The Therapeutic Perspective : Medical Practice, Knowledge, and Identity in America (Cambridge, Harvard University Press, 1986).

2. Jacques Léonard, La médecine entre les pouvoirs et les savoirs (Paris, Aubier Montaigne, 1981), 126-127. 
vait qu'induire en erreur quant aux propriétés thérapeutiques des drogues, il participe à la découverte de plusieurs alcaloïdes et introduit, en 1821, son Nouveau formulaire fondé uniquement sur des substances pures. Dans les années 1860 , beaucoup de progrès auront déjà été réalisés en pharmacologie expérimentale, incluant une meilleure utilisation des animaux de laboratoire ainsi que d'instruments sophistiqués pour mesurer les paramètres physiologiques. Au cours de ces mêmes années, la chimie organique évolue également, si bien que vers 1860 , les méthodes d'extraction, de purification et d' analyse des principes actifs sont beaucoup plus au point. On sait, en outre, comment synthétiser des produits organiques ${ }^{3}$.

Qu'en est-il dès lors des attitudes des médecins québécois, et plus spécifiquement des médecins montréalais, face à la thérapeutique au cours des dernières décennies du $\mathrm{XIX}^{\mathrm{e}}$ siècle? Comment prescrivent-ils? Que prescrivent-ils? Comment se situe la réalité de leurs pratiques par rapport au discours médical qui a jusqu'ici constitué la principale source pour saisir l'utilisation de la thérapeutique au $\mathrm{XIX}^{\mathrm{e}}$ siècle? Ce sont les questions auxquelles nous cherchons réponse à travers le croisement de deux sources, l'une témoignant des pratiques de prescription des médecins montréalais et l'autre, de leur discours sur l'usage de la thérapeutique entre 1869 et $1890^{4}$.

\section{LE MÉDICAMENT COMME PHÉNOMÈNE SOCIAL ET CULTUREL}

Le médicament est une lunette extrêmement intéressante pour regarder et analyser les pratiques et les valeurs d'une société à un moment donné. Comme objet d'étude, le médicament est beaucoup plus qu'une simple donnée médicale. Il traduit de véritables phénomènes de société. On peut penser à l'expansion considérable, depuis quelques années, de la prescription de Ritalin aux enfants en Amérique du Nord. Thème d'actua-

3. Wöhler réussit à synthétiser l'urée dès 1828. Concernant les progrès scientifiques à cet égard voir E. H. Ackerknecht, op. cit.; Olivier Faure, Histoire sociale de la médecine (Paris, Anthropos, 1994).

4. Cet article s'inscrit dans le cadre d'une vaste recherche subventionnée conjointement par le Conseil de recherche en sciences humaines du Canada et par le Hannah Institute for the History of Medicine, qui comporte trois grands axes. Le premier concerne l'évolution de l'arsenal thérapeutique en usage dans la seconde moitié du XIX ${ }^{\mathrm{e}}$ siècle et sert à identifier quelles sont les substances les plus couramment utilisées pour soigner les patients. Le second axe porte sur les pratiques de prescription des médecins et la diffusion de l'innovation scientifique en matière de thérapie médicamenteuse. Nous examinons dans quelle mesure et à quel rythme elles s'intègrent aux pratiques quotidiennes de la médecine. Nous voulons également savoir si cette intégration est uniforme à l'intérieur du corps médical montréalais ou si elle privilégie un trajet particulier à travers des réseaux d'influence ou de savoirs. Un troisième axe, enfin, est plutôt centré sur l'évolution de la pharmacie, du rôle que tiennent les pharmaciens dans la préparation des médicaments, ainsi que sur l'émergence de l'industrie pharmaceutique. 
lité, le médicament n'en est pas moins porteur de significations quant aux évolutions moins récentes qui marquent nos sociétés occidentales.

Dans le Québec de la seconde moitié du XIX ${ }^{\mathrm{e}}$ siècle, le médicament constitue un observatoire idéal pour appréhender des processus telles l'industrialisation et la commercialisation; pour capter en somme les premiers balbutiements de la société de consommation de masse telle que nous la connaissons aujourd'hui. Plus que tout autre produit, les médicaments sont prompts à entrer dans le collimateur de la transformation industrielle et commerciale. Ils sont aussi rapidement promus et célébrés comme biens de consommation dans la presse populaire et médicale. C'est du moins le témoignage éloquent que nous fournissent les pages des journaux de cette époque ${ }^{5}$. Dès la seconde moitié du XIX ${ }^{\mathrm{e}}$ siècle, on assiste en effet à l'essor d'une industrie du remède secret et de la spécialité pharmaceutique. Dans ce contexte, les médicaments sont autant perçus comme des biens de consommation susceptibles d'améliorer un quotidien parfois difficile, que comme des outils thérapeutiques destinés à contrer la maladie.

Les médicaments nous éclairent de plus sur le processus de médicalisation de la société qui s'amorce alors. Par médicalisation, il faut entendre ici le processus menant à la mise en place de moyens d'encadrement sanitaire des populations, l'instauration de mesures et éventuellement de structures de santé publique ainsi que le développement des professions de la santé. La consommation de médicaments constitue, en effet, un vecteur essentiel de ce processus. Le recours aux remèdes de tous ordres a précédé de beaucoup l'habitude de consulter les médecins, ou autres professionnels en titre, lors de la survenue d'une maladie ou d'un malaise. Tradition plus que séculaire, l'utilisation de remèdes d'inspiration populaire se transforme peu à peu, au cours de la seconde moitié du XIX ${ }^{\mathrm{e}}$ siècle, en un recours à une thérapeutique plus scientifique, transitant désormais de plus en plus souvent par la consultation médicale et la visite en pharmacie.

Nombre d'auteurs ont interprété ce phénomène comme le résultat d'une vaste entreprise d'acculturation au terme de laquelle les populations avaient été amenées à avoir recours à l'expertise médicale dans leur gestion de la maladie. À travers ce processus, les cultures populaires auraient en somme troqué leurs représentations et leurs attitudes traditionnelles face à la maladie au profit de celles imposées par l'État et par le corps médical. Cependant, on peut proposer, à l'instar d'Olivier Faure, une autre interpré-

5. Johanne Collin et Denis Béliveau, Histoire de la pharmacie au Québec (Montréal, Musée de la pharmacie, 1994); pour une période plus tardive, voir également l'ouvrage de Denis Goulet, Le commerce des maladies (Québec, Institut québécois de recherche sur la culture, 1987). 
tation du processus de médicalisation et du recours plus extensif aux soins et aux professionnels de la santé dans la seconde moitié du XIX ${ }^{\mathrm{e}}$ siècle. La médicalisation n'aurait pas été imposée «d'en haut », mais serait plutôt le résultat de la dialectique entre l'élaboration d'un projet médical soutenu par l'État — projet essentiellement hygiéniste (prévention, hygiène de vie, vaccination, etc.) - et l'expression d'une demande sociale tout entière tournée vers le curatif et vers le médicament ${ }^{6}$.

Vecteur interprétatif extrêmement sensible des attentes populaires face à la médicalisation, le médicament aurait dès lors constitué une sorte d'interface culturelle entre le projet tel que pensé par le corps médical, et les pratiques d'une population habituée de se soigner par ses propres méthodes et réfractaire à la médecine scientifique?

\section{HISTORIOGRAPHIE ET PROBLÉMATIQUE}

À ce jour cependant, peu de chercheurs se sont attachés à explorer, dans cette perspective, les aspects sociaux et culturels du recours aux médicaments. Si on recense quelques études portant sur l'histoire sociale de la pharmacie ${ }^{8}$ et d'autres sur le remède secret ou remède breveté (patent medicine) — selon des appellations qui varient d'une société à une autre - le portrait de l'évolution de la consommation de médicaments demeure toutefois encore bien parcellaire?

6. O. Faure, La médicalisation de la société dans la région lyonnaise au XIXee siècle (18001914), thèse pour le doctorat d'État, Université Lumière Lyon II, Lyon, 1989.

7. O. Faure, Les Français et leur médecine au XIX siècle (Paris, Éditions Belin, 1993).

8. Edward Kremers, Kremers and Urdang's History of Pharmacy (Philadelphie/Toronto, Lippincott, 1976, quatrième édition; Sydney W. F. Holloway, Royal Pharmaceutical Society of Great Britain 1841-1991. A Social and Political History (London, The Pharmaceutical Press, 1991); O. Faure, « Le succès du médicament en France au XIX ${ }^{\mathrm{e}}$ siècle et ses significations », dans Jean-Claude Beaune, dir., La philosophie du médicament (Paris, Champ Vallon, 1993), 216-225; O. Faure, « Une pharmacie lyonnaise et ses clients à la veille de la Première Guerre mondiale », Revue d'histoire de la pharmacie, 39,294 (1992) : 307-314. Pour le Québec et le Canada : J. Collin et D. Béliveau, op. cit.; J. Collin, Changement d'ordonnance (Montréal, Boréal, 1995); J. Collin, « Genèse d'une profession : les pharmaciens au Québec au XIX ${ }^{\mathrm{e}}$ siècle », Bulletin canadien d'histoire de la médecine, 14,2 (1997) : 241-262; Terry M. Romano, « Professional Identity and the Nineteenth-Century Ontario Medical Profession », Histoire sociale/Social History, 28,55 (1995) : 77-98; R. J. Clark, « Professional Aspirations and the Limits of Occupational Autonomy : The Case of Pharmacy in Nineteeth-Century Ontario », Bulletin canadien d'histoire de la médecine, 8,1 (1991) : 43-64.

9. Quelques recherches ont pris pour objets les remèdes secrets ou patent medicines, les percevant d'abord et avant tout comme des émanations des représentations et des pratiques populaires. La législation, la publicité, la négociation entre profanes et professionnels autour de la commercialisation de ces produits ont notamment apporté quelques indices sur une dimension non négligeable du phénomène de l'automédication. Sur les différents aspects concernant les remèdes secrets, voir John K. Crellin, Home Medicine : the Newfoundland Experience (Montréal, McGillQueen's University press, 1994); J. Collin, «Les demandes de mise en vente de médicaments en 
Au total, nous en savons davantage sur le phénomène d'automédication et sur les usages des remèdes secrets, à travers les indices que nous fournit l'abondante publicité qui les concerne, que sur le recours à l'arsenal thérapeutique tel que prescrit et utilisé par les médecins. Rares sont les études qui, à ce jour, ont pu se fonder sur des témoignages directs, afin de cerner les usages que font les médecins des médicaments pour soigner leurs patients. Le plus souvent, les auteurs se contentent de dresser un portrait impressionniste de la thérapeutique médicamenteuse prescrite, fondé généralement sur ce qu'en livre la presse médicale. Ce faisant, ils ont tendance à simplifier à l'extrême un paysage pharmaceutique et médical beaucoup plus complexe qu'il n'y paraît. Ils ont également tendance à enfermer le processus de recours aux substances médicamenteuses dans un schéma évolutif rigide et linéaire, selon lequel l'usage de l'arsenal thérapeutique progresserait tout simplement au gré de l'avancement des connaissances et de la découverte de nouvelles substances.

Pourtant, certains auteurs ont remis en question cette interprétation. Loin d'une évolution linéaire, le rapport aux médicaments connaîtrait plutôt des changements cycliques assez peu tributaires du développement des connaissances ${ }^{10}$. Lorsque l'on appréhende l'usage de la thérapeutique sur plusieurs siècles, les périodes d'utilisation forte, de polypharmacie et d'hyperactivité thérapeutique alterneraient avec les périodes de plus faible utilisation. Il se peut, en fait, que ces attitudes diverses et opposées cohabitent à toutes les époques. Ce qui changerait plutôt, d'une époque à l'autre, c'est le paradigme dominant, celui-ci variant en fonction de la position relative des différents groupes sociaux en mesure d'imposer leur rationalité. Ce sont dès lors les dynamiques sociales qui conditionneraient les évolutions tangibles dans la thérapeutique.

Par ailleurs, l'évolution scientifique en matière de médication se compliquerait également de par la trajectoire cyclique des substances ellesmêmes. Ackerknecht perçoit ainsi des cycles dans la « carrière » de plusieurs médicaments; ceux-ci commenceraient par être utilisés comme des

France à la fin du $\mathrm{XIX}^{\mathrm{e}}$ siècle », Cahiers du Centre de recherche historique de l'École des hautes études en sciences sociales, 12 : 33-51; Matthew Ramsey, «Property Rights and the Right to Health : The Regulation of Secret Remedies in France, 1789-1851 », dans William F. Bynum and Roy Porter, dir., Medical Fringe and Medical Orthodoxy, 1750-1850 (London, Croom Helm, 1987); D. Goulet, op.cit.; Ernst Stieb, « Drug Adulteration : Detection and Control in Canada. 1 : Beginnings : the Inland Revenue Act of 1875 », Pharmacy in History, 14,1 (1972), et « Drug Adulteration : Detection and Control in Canada. A Step Forward : The Adulteration Act of $1884 »$, Pharmacy in History, 18 (1976) : 17-24; James H. Young, The Toads Tool Millionaires : A Social History of Patent Medicines in America Before Federal Regulation (Princeton, Princeton Universty Press, 1961).

10. J. H. Warner, The Therapeutic Perspective : Medical Practice, Knowledge, and Identity in America (Cambridge, Harvard University Press, 1986). 
spécifiques pour devenir ensuite de véritables panacées avant de retourner, au fil des ans, à leur statut de spécifique. La quinine constitue d'ailleurs un exemple éloquent de ce phénomène. Figurant parmi les premiers alcaloïdes isolés au début du XIX ${ }^{\mathrm{e}}$ siècle, elle sera d'abord utilisée comme spécifique contre la malaria. Toutefois, on la considérera progressivement comme une substance capable de guérir des affections aussi nombreuses que disparates. Considérée comme une véritable panacée dans les années 1850-1870, elle retournera vers la fin du siècle à un destin plus modeste. La digitaline et l'iodine connaîtront également de semblables destinées ${ }^{11}$.

Enfin, une autre dimension doit être prise en compte dans l'évolution de la thérapeutique et de ses usages. Le quand, le comment et le pourquoi des changements d'attitude des médecins face à la médication ne constituent en effet qu'une partie de cette question complexe. Il faut également savoir dans quelle mesure le discours sur la thérapeutique, que permet de capter la presse médicale, s'articule aux pratiques des médecins. Si on connaît bien le discours d'une élite médicale française ou américaine sur ce sujet ${ }^{12}$, on sait peu de choses des pratiques réelles de prescription et de recours à la médication. Certains auteurs suggèrent que plus près on est de la pratique et plus on se reposera sur l'expérience et sur le sens commun plutôt que sur les théories et informations scientifiques ${ }^{13}$. Selon Jacques Léonard dépeignant les attitudes des médecins français :

[...] la tonalité générale c'est le pragmatisme. Les praticiens, sous le fouet de l'urgence, récusent la monnaie de singe des idéologies et panachent des thérapeutiques polymorphes. Les échecs sont aussi utiles que les réussites [...] mais le risque est mince d'aboutir au nihilisme thérapeutique [...] il reste courant d'accumuler les recours pour multiplier les chances de guérison. On ne sait jamais comment le traitement neutralise le mal, peu importe, on fait confiance à ce qui a coutume de réussir. L'homme de l'art ne s'occupe pas du pourquoi, il fixe son attention sur les effets. Les prescriptions sont des réponses ponctuelles à des symptômes successifs ou simultanés. Les

11. E. H. Ackerknecht, op. cit., 417. Un autre exemple du phénomène est également fourni par Jacalyn Duffin et Pierre René, « Anti-moine; Anti-biotique : The Public Fortunes of the Secret Properties of Antimony Potassium Tartrate (Tartar Emetic) », Journal of the History of Medicine \& Allied Sciences, 46,4 (1991) : 440-456.

12. Voir notamment J. Léonard, op. cit; J. H. Warner, « Therapeutic Explanation and the Edinburgh Bloodletting Controversy : Two Perspectives on the Medical Meaning of Science in the Midninetenth Century », Medical History, 24 (1980) : 241-258; J. H. Warner, «The Selective Transport of Medical Knowledge : Antebellum American Physicians and Parisian Medical Therapeutics », Bulletin of History of Medicine, 59 (1985) : 213-231; J. H. Warner, « Remembering Paris : Memory and the American Disciples of French Medicine in the $19^{\text {th }}$ Century », Bulletin of History of Medicine, 65 (1985) : 301-325.

13. Voir E. H. Ackerknecht, op. cit. et J. Léonard, op. cit. 
médecins font flèche de tout bois et continuent d'honorer la gamme multicolore des révulsifs, sédatifs, purgatifs, laxatifs, vomitifs, vermifuges, expectorants, diurétiques, sodorifiques, émollients, stimulants, narcotiques, antispasmodiques, etc., qu'offre la tradition phytothérapique et qu'enrichit l'arsenal chimique ${ }^{14}$.

Ainsi pour Léonard, le décalage existerait bel et bien entre les attitudes prônées par les grands théoriciens qui font école et la pratique quotidienne du médecin moyen. La question est peut-être de savoir si un tel fossé existe vraiment, si les positions de chacun sont aussi éloignées que ce qu'il en dit. Il est vrai que la profession médicale en France est considérablement plus structurée et hiérarchisée qu'elle ne l'est au Québec.

Mais y a-t-il rupture nette entre les théories émises par une certaine élite et le pragmatisme du praticien de la base? Ce hiatus ne révèle-t-il pas plutôt d'un temps de latence entre la diffusion d'un discours ou d'une théorie et sa retranscription dans les pratiques quotidiennes? Cette perspective soulève la question des chemins qu'emprunte l'innovation. Des auteurs ont mis de l'avant l'importance de cette dimension. Pour le cas de la France, Faure suggère ainsi plutôt que les théories médicales des premières décennies du XIX ${ }^{\mathrm{e}}$ siècle sont moins structurées et opposées qu'on ne le dit souvent et se dissolvent progressivement au fur et à mesure que l'on s'éloigne du lieu et de la date de leur conception ${ }^{15}$. Il y aurait donc une sorte de gradation dans les pratiques face à l'innovation thérapeutique.

Actuellement, rares sont les études qui permettent de répondre à ces questions, ne serait-ce que partiellement. Certaines ont permis d'affleurer cette réalité et de révéler tout l'intérêt de scruter les pratiques quotidiennes des médecins dans cette perspective ${ }^{16}$. À travers la mise en relation de la trajectoire professionnelle et des pratiques journalières d'un médecin donné, elles alimentent, par leurs conclusions, le bien-fondé de ces hypothèses, sans toutefois pouvoir les confirmer. Chaque cas demeure en effet singulier, les fonds d'archives privés de médecins étant rarissimes.

À partir de l'analyse des données d'un ordonnancier qui couvre une cinquantaine d'années d'usage de la médication, nous pensons pouvoir contri-

14. J. Léonard, op. cit., 126.

15. O. Faure, Histoire sociale de la médecine, op. cit.

16. Voir Jean-Pierre Goubert, Médecins d'hier, médecins d'aujourd'hui : le cas du docteur Lavergne (Paris, Publisud, 1992); J. Duffin, Langstaff: A Nineteenth-Century Medical Life (Toronto, University of Toronto Press, 1993); J. Duffin, « Soldiers' Work; Soldiers' Health », Labour/Le travail, 27 (1996) : 37-79; P. I. Crellin et J. K. Crellin, dir., By the Patient and not by the Book: Constancy and Change in Small Town Doctoring : Based on the Recollections of Dr. I. Hayden Lutterloh (Durham, N.C., Acorn Press, 1988); Pierre Dufour et A. Larocque, Un médecin de campagne au XX siècle (Ottawa, Musée national de l'Homme, 1985). 
buer à lever partiellement le voile sur ces pratiques. Ce portrait des pratiques de prescription de médecins montréalais sera également mis en relation avec les attitudes face à la thérapeutique que prône la presse médicale de l'époque.

\section{ENTRE DISCOURS ET PRATIQUE REGARD CROISÉ SUR DEUX SOURCES}

L'ordonnancier du pharmacien Henry R. Gray, conservé à la Faculté de pharmacie de l'Université de Montréal, constitue une source exceptionnelle pour témoigner des pratiques de prescription des médecins montréalais. Il s'agit en fait du registre où sont retranscrites intégralement les ordonnances médicales exécutées par le pharmacien dans l'exercice de ses fonctions. Cet ordonnancier se compose d'une cinquantaine de volumes (comprenant chacun de 300 à 600 pages), comportant plus de 175000 ordonnances s'échelonnant sur toute la durée de sa vie professionnelle, soit sur près d'un demi-siècle (1859-1907).

Son propriétaire, le pharmacien H. R. Gray, occupe tout au long de sa carrière des fonctions importantes, tant dans le domaine de la santé publique que dans celui de l'organisation de la profession pharmaceutique. Ces fonctions rendent donc plus facile le repérage de son itinéraire. À la fois catholique et anglophone, H. R. Gray figure parmi les militants les plus actifs du processus menant à la reconnaissance légale de la profession à compter de la fin des années 1860. Président de l'Association pharmaceutique de la province de Québec de 1875 à 1877, et, de nouveau, entre 1891 et 1895, il cumule ces charges avec celle de président de la première institution d'enseignement de la pharmacie, le Montreal College of Pharmacy au cours des années $1860^{17}$. Il devient en outre président du Comité d'hygiène de la ville de Montréal en 1885, alors qu'éclate une importante épidémie de variole à Montréal ${ }^{18}$. Parallèlement à cette carrière d'homme public, il tient pharmacie pendant cinquante ans au n ${ }^{\circ} 122$ de la rue Saint-Laurent, pratique dont témoigne l'ordonnancier. Bien situé, son commerce enregistre un débit fort important de marchandises. Si l'ordonnancier ne saurait traduire un portrait exhaustif de la pratique de chacun des prescripteurs, puisque leurs patients sont libres de se présenter à la pharmacie de leur choix, il n'en concerne pas moins les prescriptions d'une proportion considérable des médecins exerçant à Montréal à cette époque ${ }^{19}$.

17. Voir J. Collin et D. Béliveau, op. cit.; également le Dictionnaire biographique du Canada, XIII.

18. Voir Michael Bliss, Montréal au temps du grand fléau. L'histoire de l'épidémie de 1885 (Montréal, Libre expression, 1993).

19. Le total regroupe en fait 143 médecins inscrits dans l'ordonnancier en 1869 et 219 inscrits en 1890. Pour chacune de ces années, les prescripteurs de l'ordonnancier représentent la quasitotalité des praticiens montréalais de l'époque selon le croisement de différentes sources, à savoir les 
La longue durée de cette source, l'uniformité du type de données qui y sont consignées, l'importance de la pharmacie et la notoriété de son propriétaire sont autant d'éléments qui en rendent l'analyse pertinente, tout en étant garants d'une certaine représentativité et d'une portée heuristique indéniable.

Quant à la presse médicale, elle a fait l'objet du dépouillement systématique des rubriques concernant la thérapeutique et la matière médicale. Pour ce faire, nous nous sommes centrées sur les principales revues anglophones et francophones de l'époque, dont la plupart sont, du reste, publiées à Montréal $^{20}$. Plusieurs consacrent régulièrement quelques pages à ce thème. Mentionnons, notamment, les rubriques « The Past Year », du Canada Lancet en 1864-1865, « Therapeutics », du docteur J. Stewart dans le Canada Medical and Surgical Journal à compter de 1881 ainsi que celle qui s'intitule "Thérapeutique », sous la responsabilité du docteur H. E. Desrosiers, dans l'Union médicale du Canada à compter de 1882. D'autres rubriques plus générales, comme celle du Canada Medical Record intitulée "Progress of Medical Science », dans les années 1880, contiennent également des informations pertinentes concernant la thérapeutique. La presse médicale permet ainsi de pressentir les attitudes du corps médical face à la thérapeutique, de déceler la présence ou non de courants dominants à cet égard chez les médecins québécois et montréalais, d'éclairer et éventuellement de confronter ces discours avec les pratiques qui se dégagent de l'analyse de l'ordonnancier.

\section{LE DISCOURS DES MÉDECINS MONTRÉALAIS FACE À LA THÉRAPEUTIQUE}

On ne saurait parler d'un seul discours, bien campé et univoque, portant sur la thérapeutique et l'usage de la matière médicale entre 1869 et 1890. Non pas que plusieurs écoles de pensée s'affrontent. Il s'avère plutôt qu' aucune ne retient l'adhésion des auteurs. La période s'inscrit, semble-t-il, d'une façon assez manifeste, dans une mouvance qui favorise un certain rationalisme expérimental en matière de thérapeutique ${ }^{21}$ et qui rejette tout autant le dogme que l'habitude et les solutions toutes faites lorsqu'il s'agit de prescrire une médication.

recensements de 1871 et de 1891 , les Lovells ainsi que les registres du Collège des médecins et chirurgiens de la province de Québec.

20. Nous avons ainsi dépouillé les revues suivantes pour la période allant du début des années 1860 jusqu'en 1890, en fonction, il va sans dire, des dates de parution de ces journaux : Canada Lancet (1863-1864); Canada Medical Journal and Monthly Record of Medical and Physical Science (1864-1872); Canada Medical Record (1872- ); Canada Medical and Surgical Journal (1872- ); L'Union médicale du Canada (1872- ).

21. Le terme désigne une démarche conciliant raisonnement et expérimentation. 
Au milieu des années 1870, un article du docteur Canniff de Toronto exhorte les médecins à faire preuve de plus de discernement dans leur recours à l'arsenal thérapeutique et à ne pas se laisser guider par l'unique désir d'agir et de répondre aux attentes des patients en quête d'une solution concrète et tangible à leurs problèmes de santé. Selon lui, le médecin n'est pas seulement un distributeur de médicaments. Il doit faire confiance à la nature dans les stratégies thérapeutiques qu'il élabore. "The knowledge requisite is not only to enable him to do what is necessary, but to avoid that which is unnecessary », explique-t-il. « The administration of bread pills and tinctured water is one of the clumsy ways of solving the question. But apart from this morbid desire on the part of the public and the expectations that medicine will be given, does not the doctor sometimes magnify his office by unnecessary service ${ }^{22}$ ?

Le docteur Canniff est-il seul à prôner un certain scepticisme face au médicament? Un article, beaucoup plus récent (1929) mais évoquant cette période, suggère que l'attitude était répandue chez les médecins québécois de l'époque. Lors du décès du docteur L.-D. Mignault, une rubrique nécrologique évoque les années 1870 en ces termes : «Le professeur Mignault a vécu à une époque de nihilisme thérapeutique. On faisait alors un diagnostic soigné, on établissait un pronostic sérieux, puis, sous prétexte que l'on ne pouvait s'attaquer à la cause de la maladie ou que le mal était incurable, on prescrivait un traitement quelconque et on laissait à la nature, le soin de guérir le malade ${ }^{23}$. »

Pourtant, dès le milieu des années 1870, plusieurs articles émanant de la presse médicale évoquent plutôt une renaissance de la thérapeutique et prêchent en faveur de sa revalorisation. C'est ainsi qu'on pourra lire en éditorial du Canada Lancet en 1874 :

Ten or twenty years ago the chief line of medical investigation was pathology. The fascination of this study still holds ground; and a vast accumulation of facts and knowledge, the pathological work of our time, has served to place the practice of medicine and surgery on a more thoroughly scientific foundation. Now, howerver, there is to be noticed a strong tendency towards therapeutics. This branch, too much neglected by the pathologists, now comes back to be held in something

22. William Canniff, « Nature's Power to Heal », CMR, 4 (1875) : 2. Il s'agit d'une intervention de W. Canniff devant l'Association médicale canadienne. Ce scepticisme face aux médicaments n'est peut-être pas sans rapport avec ses positions mieux connues face au listerisme. À ce sujet, voir Jim T. Connor, «Listerism Unmasked : Antisepsis and Asepsis in Victorian Anglo-Canada », Journal of the History of Medicine \& Allied Sciences, 49,2 (1994) : 207-239.

23. UMC, 58 (1929) : 590. 
like the same importance it had in the early days of medicine, as was need the case when practice was solely based upon empiricism ${ }^{24}$.

D'ailleurs, les médecins francophones semblent également faire écho à cette tendance. En 1878, le docteur Lachapelle se positionne contre une attitude expectante et prône la réhabilitation de la thérapeutique. Prenant prétexte d'un article intitulé «Quelques considérations sur la thérapeutique au sujet de la médication alcoolique dans la pneumonie », Lachapelle exhorte ses collègues à se défier du scepticisme thérapeutique qu'il identifie comme le courant dominant ${ }^{25}$. Il perçoit cette attitude comme le résultat de la multiplication des écoles de pensée et des tendances thérapeutiques souvent contradictoires et éphémères qui ont marqué le $\mathrm{XIX}^{\mathrm{e}}$ siècle. Du reste, il comprend d'autant mieux l'attitude de ses collègues, qu'il constate lui-même que si les certitudes s'accumulent dans le domaine du diagnostic, elles n'en rendent que plus frappant le maintien des impuissances en matière de thérapeutique. Il faut toutefois s'éloigner, selon lui, des dogmes pour s'orienter davantage vers une démarche d'expérimentation. Il propose ainsi de «chercher ailleurs que dans le rationalisme médical absolu les éléments qui doivent régénérer la thérapeutique [...] » et développe : «Telle doit être la sage direction donnée à la thérapeutique : interrogeons d'abord l'empirisme; demandons-lui des faits; expérimentons-les à notre tour et le dogmatisme viendra à la fin; et si nous ne pouvons dogmatiser, nous nous consolerons facilement de ses idéalités par un succès plus fréquent. » Compte tenu du développement de nouvelles substances et de leur utilisation, il ajoute que la thérapeutique est en droit de réclamer davantage de positivisme ${ }^{26}$.

Quelle que soit la place consentie par les médecins à l'usage des médicaments en ce début des années 1880 au Québec, certains signes, telle la remise en question de l'enseignement de la thérapeutique, font la preuve d'un regain d'intérêt pour la branche. On note la séparation des cours de matière médicale et de thérapeutique dans plusieurs facultés de médecine, ainsi que la parution de plusieurs éditoriaux abordant cette question dans les revues montréalaises.

24. The Canada Lancet, 7 (1874).

25. UMC, 7 (1878) : 241-246, 289-295.

26. Ce faisant, il s'inscrit dans un courant que défendent également à la même époque certains de ses collègues français. Voir C. Bouchard, « La méthode en thérapeutique », UMC, 9 (1880) : 402-409, 440-445, 489-496. L'auteur est professeur de pathologie et de thérapeutique générales à la Faculté de médecine de Paris. 
Dans un éditorial publié en août 1882 et intitulé simplement « Thérapeutique vs. matière médicale », l'auteur souligne l'importance de faire la distinction entre les deux termes ${ }^{27}$. Selon lui ${ }^{28}$, telle qu'enseignée, la matière médicale

se borne le plus souvent à traiter des propriétés physiques et des caractères botaniques des médicaments [...] insistant aussi peu que possible sur l'action physiologique et thérapeutique. En outre, on semble se plaire très souvent à y énumérer au long un grand nombre de formules officinales et magistrales et l'on force les élèves à apprendre ces formules, comme si la thérapeutique pouvait se renfermer tout entière dans une ordonnance. En deux mots, on donne un cours de pharmacologie ${ }^{29}$.

Ce cours, utile pour les pharmaciens de dire l'auteur, ne saurait répondre aux besoins des futurs médecins. Ce qu'ils doivent apprendre et connaître, c'est l'action physiologique des remèdes, les usages et les doses à prescrire, c'est donc l' « art thérapeutique ${ }^{30} »$.

À une autre occasion, le docteur N. Fafard souligne d'ailleurs le chemin parcouru, sur le plan des connaissances :

Il y a à peine 20 ans, [...] on n'étudiait les affections morbides que par leurs symptômes : de là le choix des médicaments ne pouvant se faire que d'après les données de l'expérience. Impossible de se rendre compte du modus operandi de la médication instituée. Tel remède a été bon dans telle maladie, il doit l'être encore dans cette même maladie : voilà le raisonnement que l'on se faisait; le succès donnait la vogue au médicament et on l'employait $[\ldots]^{31}$.

La science, et notamment l'histologie et la chimie, auraient, selon Fafard, balayé ces tendances et les écoles thérapeutiques en découlant :

Doit-on s'étonner maintenant que les préparations mercurielles soient si efficaces dans les maladies syphilitiques; que l'iode absorbé par les lymphatiques fasse disparaitre l'engorgement glandulaire; que la quinine, à haute dose et donnée à temps voulu, c'est-à-dire plusieurs heures avant l'attaque, empêche la reproduction des germes et par là

27. UMC, 11 (1882) : 404-405.

28. II s'agit probablement de Desrosiers qui est à l'époque l'un des rédacteurs de la revue avec Lamarche et Lachapelle et qui se préoccupe tout particulièrement de ces questions.

29. Ibid., 404.

30. Quelques mois auparavant, le docteur L. J. V. Cléroux, s'exprimant devant la Société médicale de Montréal, soutenait de semblables propos, montrant finalement que certains médecins avaient déjà intégré cette nouvelle vision « revalorisante » de la thérapeutique. Voir L. J. V. Cléroux, «Considérations générales sur la physiologie du médicament », UMC, 11 (1882) : 244-253, 327-333.

31. N. Fafard, «Étude sur les fermentations », UMC, 10 (1881) : 97-104. Il s'agit d'une communication devant la Société médicale de Montréal. 
même les symptômes qui l'accompagnent? Il est facile aussi de s'expliquer pourquoi l'onguent de calomel et l'onguent de broxide de mercure agissent si bien contre la teigne, le prurigo, le rifle etc. [...]. On peut maintenant, jusqu'à un certain point, expliquer l'action de toute cette classe de médicaments altérants qui sont tous des poisons plus ou moins énergiques et que l'on emploie d'habitude contre les maladies contagieuses. Aujourd'hui on prouve qu'ils sont bons parce qu'ils sont des poisons et qu'en cette qualité ils détruisent les germes, cause immédiate de toutes ces maladies restées si longtemps ignorées ${ }^{32}$.

Compte tenu de ces développements dans les connaissances, mais également de la complexité croissante d'un paysage pharmacologique en constant réaménagement, il faut développer une attitude positive ainsi qu'une démarche scientifique alliant le raisonnement à l'expérimentation dans le recours à un arsenal thérapeutique dont l'exercice de la médecine ne saurait désormais se passer.

On ne prescrit plus avec, comme seuls objectifs, l'éradication des symptômes et la stimulation des capacités de guérison du patient. L' '«art de la thérapeutique » est en soi fort complexe. Exposant régulièrement ses vues dans le cadre d'une rubrique de L'Union médicale du Canada sur la « Thérapeutique générale », le docteur H. E. Desrosiers affirme ainsi que « dans la thérapeutique de toute maladie, nous avons à considérer d'abord la nature de l'affection elle-même; en second lieu la nature des accidents liés à cette affection ${ }^{33}$ ». Prenant en exemple l'un de ses cas cliniques, il ajoute :

Ici nous avions à combattre une maladie : la néphrite, un symptôme : l'urémie, un accident : les convulsions. Évidemment il fallait courir au plus pressé. Les convulsions étant essentiellement liées à l'intoxication urémique, il était urgent de songer aux moyens de favoriser l'élimination de l'urée hors du système, sauf à diriger ensuite ou concurremment notre thérapeutique contre la lésion rénale. Mais je le répète, ce qu'il importait de faire c'était de combattre l'urémie et, par le fait même, ses manifestations redoutables : médication toute symptomatique ${ }^{34}$.

Le choix de la médication reposerait donc notamment sur deux choses : le traitement de la maladie en tant « qu'essence morbide » (traitement pathogénique) et le traitement des accidents (traitement symptomatique) ${ }^{35}$.

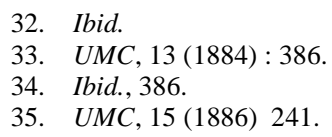


Ce nouveau regard sur la thérapeutique incline à penser que les années 1870 et 1880 ne sont pas celles d'un scepticisme généralisé. Toute nouvelle substance s'avère présenter un potentiel qu'il s'agit d'explorer à travers des tests de laboratoire et ce que l'on pourrait assimiler aux premiers « essais cliniques ${ }^{36} »$. L'attention ne se limite toutefois pas aux nouveaux médicaments. La période semble en effet marquée par l'utilisation à de nouvelles fins thérapeutiques de substances déjà connues ${ }^{37}$. On pressent, ainsi, avec une sensibilité particulière toute la gamme des possibilités thérapeutiques que peut offrir une même substance administrée sous une forme différente et à des doses variables. En effet, selon l'un des rédacteurs de la revue The Canada Lancet:

As regards the materia medica, progress takes two lines, one in the direction of adding new medicamenta to the list, the other in finding out new properties in the older remedies, and in more accurately defining the methods of operation and the modus curandi of medicines. To cite but an instance or two by way of suggestion. How very important it is to know that, in suitable doses, digitalis, instead of acting as a sedative and depressant, improves the nutrition of the heart, strengthens its muscular walls, and gives tone to the nervecenters presiding over this viscus. And yet this is new knowledge of an old medicine ${ }^{38}$.

En fait, ce n'est pas tant la substance en elle-même que la façon dont on la met à contribution (dosage, combinaison à d'autres substances, etc.) dans la thérapie qui en permet l'efficacité. Ainsi, dans les cas où il n'existe pas encore de spécifiques, écrit le docteur Desrosiers dans l'une de ses rubriques, c'est-à-dire dans la majorité des cas à traiter, il est primordial de prendre en compte le malade pour éviter d'être un «médecin à tiroirs ». Il désigne par là l'attitude voulant que l'on traite tous les patients souffrant d'une même affection par les mêmes moyens ${ }^{39}$. Que faut-il alors prendre en compte? L'âge, le sexe, la constitution, les habitudes (alcoolisme, diathèses), sans oublier la douleur; d'où l'emploi

36. Ainsi Desrosiers s'inspire apparemment beaucoup de recherches et d'expériences françaises, pour la plupart consignées dans les travaux de la Société de thérapeutique de Paris pour établir ses récapitulatifs annuels. Selon lui, « la Société de thérapeutique se tient constamment à la tête du mouvement thérapeutique en France et tous les médicaments nouveaux y sont étudiés et expérimentés avec une attention scrupuleuse qui ne laisse pas place à l'enthousiasme exagéré de certains expérimentateurs étrangers». UMC, 18 (1889) : 62.

37. Voir à titre d'exemple, la "Conversation des drogues ", poème illustrant le caractère réversible de l'abandon de telle ou telle substance, de telle ou telle méthode thérapeutique. UMC, 4 (1875) : 134-136.

38. The Canada Lancet, 7 (1874): 25-26.

39. UMC, $15(1886): 243$. 
d'analgésiques auxquels il consacre une leçon en $1886^{40}$. Il faut se débarrasser de la douleur chaque fois que possible; en enlevant d'abord la cause puis en calmant la douleur elle-même. La thérapeutique doit toujours être rationnelle. Il exhorte dès lors les médecins à raisonner leur traitement tout comme ils auront raisonné leur diagnostic.

Mais la recherche de l'efficacité de la médication n'est pas le seul élément qui structure le discours médical face à l'usage des médicaments. La crainte des empoisonnements, de plus en plus nombreux semble-t-il, conduit la presse médicale à attacher une grande importance à une nouvelle dimension de la médication : son innocuité. L'attention à cette dimension s'avère ainsi l'une des grandes nouveautés dans le domaine de la thérapeutique au cours de cette période, attention qui va prendre encore davantage d'ampleur avec les années 1880 et se manifester sous la forme d'une réduction des doses en général, mais aussi passage au fractionnement, à une posologie plus nuancée ${ }^{41}$. Elle s'accompagne d'études sur les dangers des substances et sur leur toxicité. D'où l'intérêt, très perceptible au travers de la presse médicale, pour les cas d'empoisonnement, la nécessité d'une législation réglementant la vente des «poisons », mais aussi pour la recherche d'antidotes et de substituts à certaines substances. D'où, également, l'insistance sur la nécessité d'être toujours en éveil, de suivre attentivement les progrès de la maladie et les effets d'une médication donnée sur le malade en privilégiant des thérapies complexes, toutes en nuances et en faibles dosages. Le médecin doit attribuer à son patient une importance centrale et garder en tête l'atteinte de son bien-être.

Pendant cette période, le discours perçu à travers la presse médicale va dans le sens d'une revalorisation de l'arsenal thérapeutique. Certes, on admet d'emblée que la thérapeutique permet davantage de soulager que de guérir et qu'on dispose de peu de spécifiques pour agir véritablement et efficacement contre la maladie. Mais l'heure est à l'expérimentation et au raisonnement, plutôt qu' au scepticisme thérapeutique. La revalorisation du recours à la matière médicale passe par une redéfinition de l' «art de prescrire » comme d'une opération complexe. Elle requiert la maitrise d'un savoir et d'une attitude positiviste faisant du médecin un véritable scientifique. Ces exigences nouvelles doivent se traduire par la mise en œuvre de stratégies thérapeutiques propres à chaque malade, axées sur la combinaison de substances et la modulation de leur dosage. Voyons dès lors ce qu'il

40. UMC, 15 (1886) : 336-343.

41. UMC, 16 (1887) : 163-164. 
en est des pratiques concrètes de prescription des médecins à cette même époque, à travers l'analyse des données de l'ordonnancier de H. R. Gray.

\section{L'USAGE DE LA MATIÈRE MÉDICALE CHEZ LES MÉDECINS MONTRÉALAIS}

\section{Portrait de l'arsenal thérapeutique utilisé, 1869-1890}

C'est à un paysage complexe et polymorphe que renvoie l'usage de la matière médicale dans le dernier tiers du XIX ${ }^{\mathrm{e}}$ siècle. L'image que traduit généralement l'historiographie ne saurait rendre compte de cette complexité. D'où l'intérêt de pouvoir se fonder sur une source de première main, comme celle que représente l'ordonnancier du pharmacien Gray, pour saisir les pratiques des médecins.

Tout au long de la période que couvre l'ordonnancier, soit de 1859 à 1907, les ordonnances y sont numérotées en continu. Les informations qu'elles comportent sont d'une grande uniformité en ce qui concerne la composition et la préparation des médicaments prescrits, leur forme pharmaceutique, leur posologie, le nom du médecin prescripteur et la date de l'ordonnance. Moins régulièrement, les entrées indiquent le nom du patient et le problème pour lequel il est traité (figure 1). Pour les fins du présent article, nous avons informatisé et analysé les données contenues dans l'ordonnancier concernant les années 1869 et 1890. Ces années correspondent en effet aux bornes de la période pour laquelle nous avons dépouillé la presse médicale.

L'ordonnancier nous révèle ainsi qu'en 1869, pas moins de 1815 ordonnances sont exécutées à la pharmacie de Gray, ce qui représente une moyenne de six ordonnances par jour puisque la pharmacie est ouverte tous les jours de la semaine y compris le dimanche. Quelque vingt ans plus tard, soit en 1890, le nombre des ordonnances exécutées à cette même pharmacie aura presque triplé, pour atteindre 4575, faisant foi d'une activité pharmaceutique intense (tableau 1).

Certes, nous sommes loin des formules de jadis faisant appel à des dizaines d'ingrédients. En 1869, comme en 1890, les ordonnances comportent en moyenne trois ingrédients, leur nombre pouvant néanmoins varier de un à huit. Le temps de la médecine héroïque est lui aussi bien terminé puisque l'utilisation, notamment, du calomel et du tartre émétique est très marginale.

Par ailleurs, plusieurs centaines de substances différentes font partie de l'arsenal thérapeutique utilisé par les médecins montréalais. Déjà en 
FIGURE 1

\section{L'ORDONNANCIER}

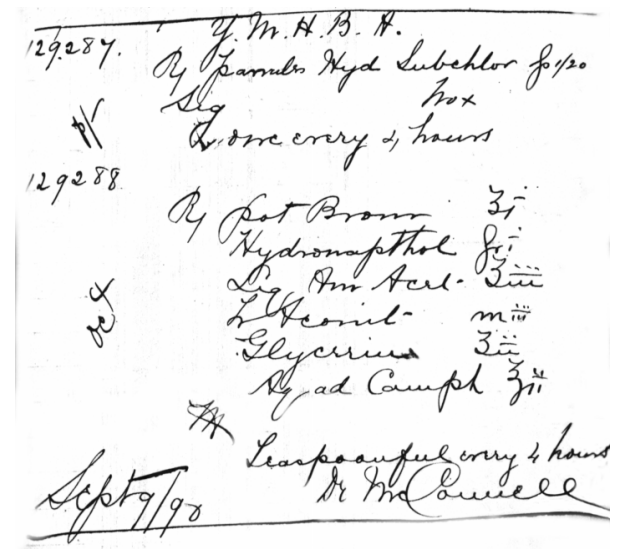

TABLEAU 1

PORTRAIT GLOBAL DE L'ORDONNANCIER 1869 ET 1890

$\begin{array}{lrr} & 1869 & 1890 \\ \text { Nombre de prescriptions } & 1815 & 4575 \\ \text { Nombre de prescripteurs } & 143 & 352 \\ \text { Nombre de substances } & 597 & 1243 \\ \text { Nombre de substances maîtresses } & 170 & 294\end{array}$

1869, époque pourtant décrite comme empreinte de scepticisme face à la thérapeutique, on dénombre 597 substances différentes entrant dans la composition des ordonnances médicales. À la fin de la période étudiée, 1243 éléments distincts y figurent, ce qui constitue un accroissement considérable (tableau 1). Cette tendance est du reste conforme à celle que suggère la presse médicale : il y aurait, d'une part, cumul de nouvelles substances et, d'autre part, réutilisation des anciennes à de nouvelles fins.

Lorsqu'il est question de matière médicale et de pharmacopée, l'historiographie renvoie le plus souvent à une classification fondée sur l'origine des substances, soit végétale, minérale ou animale. Un tel regroupement s'avère cependant anachronique et inapte à traduire la forte prépondérance de la chimie dans le paysage pharmaceutique de l'époque. Par ailleurs, autre signe des temps, une catégorisation des substances en fonction de 
leurs propriétés thérapeutiques s'avère irréaliste à une époque où l'on s'évertue à découvrir à une même substance de multiples propriétés. C'est dès lors en regard de leur nature chimique, notion relativement imperméable au passage du temps et aux changements de lieux que nous avons regroupé les substances de l'ordonnancier pour les fins de notre analyse. Cinq catégories ont été créées : les composés organiques, les composés inorganiques, les préparations, les simples et les remèdes secrets ${ }^{42}$.

Les composés inorganiques s' avèrent être des entités chimiques complexes qui sont dépourvues de carbone. On y retrouve les minéraux, tels le mercure, le plomb, le fer. Le nombre des substances s'inscrivant dans cette catégorie passe de 112 à 145 de 1869 à 1890, bien que leur poids relatif tende à diminuer d'une période à l'autre (tableau 2). Les composés organiques sont également des entités chimiques complexes mais qui contiennent du carbone, comme l'éther ou l'alcool. Ils sont moins nombreux que les composés inorganiques, bien qu'ils regroupent près d'une centaine de substances différentes. Leur nombre passe de 76 à 126 au cours de la période. La catégorie des simples inclut des plantes entières, des parties de plantes ou des substances animales, dans leur forme la plus rudimentaire ou ayant subi un traitement minimal. On remarquera que ces substances « naturelles » occupent une place nettement moins importante que les substances chimiques dans l'arsenal thérapeutique. Ce sont en fait les préparations qui regroupent le plus vaste éventail de produits, puisqu'on en dénombre plusieurs centaines ( 311 en 1869 et 615 en 1890). Ces préparations officinales, parmi lesquelles on compte les sirops, les extraits, élixirs, pilules, constituent en soi des entités pouvant contenir un amalgame de composés inorganiques, de composés organiques ou de simples. Elles sont préparées à l'avance par le pharmacien selon la formule inscrite dans l'une des pharmacopées officielles. Enfin, les remèdes secrets sont des remèdes vendus au détail sous un nom de marque, prêts-à-consommer, dont la formule est maintenue secrète. Peu nombreux parmi les ingrédients de chaque ordonnance en 1869 , leur nombre s' accroît nettement en 1890, passant de 15 à 168. Cette augmentation laisse d'ailleurs présager un recours de plus en plus fréquent à la fin du siècle.

\section{Un rapport complexe à la matière médicale}

Toutes ces substances utilisées par les médecins prescripteurs sont également susceptibles d'être regroupées selon leur appartenance à de

42. En même temps qu'il nous procurait un système de classification fonctionnel, un tel découpage permettait également de saisir le degré de manipulation requis par le pharmacien dans l'exécution des ordonnances. Les catégories de substances ont été établies notamment à partir du système de classification de C. S. Hallberg (v. 1880), directeur du National Institute of Pharmacy (Chicago). Elles correspondent également à celles utilisées par Dorvault en 1923 (L'officine ou répertoire général de pharmacie pratique). 
TABLEAU 2

\section{NOMBRE DE SUBSTANCES INSCRITES DANS L'ORDONNANCIER PAR CATÉGORIES 1869 ET 1890}

Catégorie

\section{Composé inorganique}

Composé organique

Préparation

Remède secret

Simple

Indéfinie

Total annuel
1869

1890

$\begin{array}{rrrr}\text { Nombre } & \% & \text { Nombre } & \% \\ 112 & 19 & 165 & 13 \\ 76 & 13 & 126 & 10 \\ 311 & 52 & 615 & 49 \\ 15 & 3 & 168 & 14 \\ 56 & 9 & 112 & 9 \\ 27 & 5 & 57 & 5 \\ 597 & 100 & 1243 & 100\end{array}$

grandes familles que l'on appelle les substances maîtresses. Le mercure, par exemple, constitue la substance maîtresse à partir de laquelle sont produits différents composés inorganiques tels que le biiodure de mercure, le bichlorure de mercure, le protochlorure de mercure appelé également calomel. De même, le potassium s'avère le matériau à la base des composés comme l'acétate de potassium, le bromure de potassium et le bicarbonate de potassium. L'ordonnancier de Gray permet de dénombrer 170 substances maîtresses en 1869 et 294 en 1890. Les tableaux 3 et 4 permettent de voir lesquelles de ces substances sont les plus couramment utilisées au cours des deux années qui balisent la période étudiée ici ${ }^{43}$.

Même agrégées, synthétisées et regroupées, les substances entrant dans la composition des diverses prescriptions ne sauraient, dès lors, se réduire à quelques produits, tels le calomel et le tartre émétique, ou encore à quelques grandes familles de matériau, tels l'opium, la quinine ou le fer. En fait, les dix substances maîtresses les plus fréquentes en 1869 ne sont utilisées que dans $36 \%$ des cas (tableau 3). C'est dire que près de deux fois sur trois (64\%), on a recours à des substances autres que celles généralement identifiées à travers l'historiographie. En 1890, la diversification des substances prescrites est plus grande encore puisque les dix substances maîtresses les plus utilisées ne comptent que pour $30 \%$ des utilisations (tableau 4$)^{44}$.

43. Les tableaux 3 et 4 donnent l'impression que l'utilisation de l'alcool est peu fréquente. La raison en est que cette substance figure rarement comme ingrédient spécifique dans les prescriptions. L'usage à cette époque en est néanmoins abondant puisque l'alcool entre dans la composition des nombreuses préparations officinales prescrites, telles que les vins, teintures et élixirs.

44. On trouvera d'ailleurs en annexe la liste nominale des substances maîtresses incrites à l'ordonnancier entre 1869 et 1890. 
TABLEAU 3

\section{NOMBRE D'UTILISATIONS* DES 10 PRINCIPALES SUBSTANCES MAÎTRESSES EN 1869}

Nom de la substance maîtresse

$\begin{array}{cr}\text { Nombre } & \% \\ 389 & 8 \\ 253 & 5 \\ 206 & 4 \\ 199 & 4 \\ 169 & 3 \\ 156 & 3 \\ 119 & 2 \\ 117 & 2 \\ 116 & 2 \\ 103 & 2 \\ 3257 & 64 \\ 5084 & 100\end{array}$

Potassium

Quinine

Fer

Opium 4

Mercure

Acide sulfurique

Ammonium

Ipécacuanha 2

Camphre

Vin 2

Autres 64

Total 5084 100

*Le nombre d'utilisations représente le nombre de fois où une même substance est prescrite.

\section{TABLEAU 4}

\section{NOMBRE D'UTILISATIONS* DES 10 PRINCIPALES SUBSTANCES MAÎTRESSES EN 1890}

$\begin{array}{lcc}\text { Nom de la substance maîtresse } & \text { Nombre } & \% \\ \text { Potassium } & 810 & 6 \\ \text { Fer } & 496 & 4 \\ \text { Quinine } & 405 & 3 \\ \text { Sodium } & 399 & 3 \\ \text { Indéfinie } & 347 & 3 \\ \text { Glycérine } & 340 & 3 \\ \text { Opium } & 307 & 2 \\ \text { Camphre } & 290 & 2 \\ \text { Noix vomique } & 263 & 2 \\ \text { Mercure } & 259 & 2 \\ \text { Autres } & 9324 & 70 \\ \text { Total } & 13240 & 100\end{array}$

*Le nombre d'utilisations représente le nombre de fois où une même substance est prescrite. 
Comme le suggère la presse médicale, c'est dans la variation des substances, des combinaisons et des dosages que semble se définir concrètement le rapport des médecins à la thérapeutique. Nous avons ainsi regardé avec beaucoup d'attention les «épisodes de maladie » repérés à travers l'ordonnancier et s'échelonnant parfois sur quelques jours, voire sur quelques semaines. Certains médecins indiquent en effet assez systématiquement le nom de leur patient sur l'ordonnance, d'autres ne le faisant que de manière occasionnelle. Les habitudes de prescriptions des premiers nous ont ainsi permis de repérer plusieurs épisodes de maladie.

Au cours de chaque épisode, le patient se voit prescrire quasi quotidiennement une nouvelle médication. À titre d'exemple, le cas de Mrs Grundell qui, entre le 25 juillet et le 28 août 1869 , recevra 13 ordonnances émanant en partie du docteur Hingston et en partie du docteur Perrigo. Si cet épisode soulève toute la question du recours au médecin en lui-même ${ }^{45}$, il est également assez éloquent quant au recours à l'arsenal thérapeutique. Le 25 juillet, un remède composé d'eau, de teinture de jusquiame, de vin d'ipécacuanha ainsi que d'un alcoolat ammoniacal est prescrit à la patiente par le docteur Perrigo. Une semaine plus tard, une ordonnance composée de teinture d'opium, d'un liniment au chloroforme et d'une essence de thérébentine est préparée pour elle. Elle s'accompagne, le jour même, d'une autre prescription comportant cette fois-ci un mélange de protochlorure de mercure et de bicarbonate de sodium. Le lendemain, 2 août, c'est le docteur Hingston qui prend la relève en prescrivant des pilules d'opium camphré. Le vendredi 6 août, il récidive avec une préparation composée d'eau, de sirop d'écorce d'orange amère, d'un alcoolé sulfurique et de sulfate neutre de quinine. Le lendemain, poudre d'opium et acétate de plomb remplacent ou s'ajoutent aux médicaments prescrits la veille. Cette même préparation sera prescrite trois jours d'affilé, faisant l'objet, chaque fois, d'une ordonnance chez le pharmacien Gray. Enfin, du 11 au 28 août, la patiente se voit encore prescrire cinq différents médicaments par le docteur Hingston : teinture de cachou, mixture avec de la craie et teinture de kino forment la première ordonnance, acide tannique, les deuxième et troi-

45. On peut en effet se demander pourquoi la patiente est traitée par deux médecins en alternance. Ces derniers se partagent-ils une clientèle, se remplacent-ils mutuellement lorsque l'un ne peut être présent, est-ce parce qu'il s'agit de la période estivale? En fait, le docteur Perrigo traite également le capitaine Grundell à cette même époque et il y a fort à parier qu'il en est à ses débuts dans la carrière et qu'il seconde Hingston. Il n'obtiendra en effet sa licence qu'en 1870. 
sième, liqueur de chlorhydrate de morphine et eau la quatrième, enfin suivie d'une prescription de poudre d'opium et sulfate de sodium en poudre.

Vingt ans plus tard, on note la même tendance à rédiger, au jour le jour, une ou plusieurs ordonnances destinées à la fois au traitement pathogénique ${ }^{46}$ et au traitement symptomatique de la maladie. Deux ordonnances distinctes, composées pourtant des mêmes ingrédients actifs peuvent ainsi être prescrites par le même médecin au même malade au cours d'une seule journée. À titre d'exemple, le cas d'Alexander Dougall soigné par le docteur Blackader. Il reçoit, le $1^{\text {er }}$ mars, une médication composée d'iodure de potassium, de bicarbonate de potassium et de carbonate d'ammoniaque auxquels s'ajoutent un extrait acqueux de réglisse et un sirop d'écorce d'orange amère. Une seconde ordonnance comporte les mêmes deux ingrédients actifs, mais dans des dosages différents. À ceuxci s'ajoutent de la teinture de colchique, du sirop de salsapareille composé ainsi que de l'eau. On perçoit dès lors cette extrême attention aux dosages qui, en général, sont faibles, ainsi que la modulation du traitement prescrit en fonction d'une observation attentive de l'évolution de la maladie.

En définitive, à l'instar de ce que suggère la presse médicale, les pratiques des prescripteurs de l'ordonnancier ne reflètent aucunement le scepticisme thérapeutique supposé par maints auteurs comme la réaction inévitable à plusieurs décennies d'hyperactivité en matière de thérapeutique. Le portrait esquissé ici à grands traits suggère plutôt un recours extensif à l'arsenal thérapeutique. Les données de l'ordonnancier ne permettent certes pas de saisir la rationalité qui sous-tend les pratiques. Les médecins font-ils preuve de ce mélange de raisonnement et d'expérimentation auquel les exhortent leurs pairs s'exprimant à travers la presse médicale? Leur recours à la thérapeutique est-il davantage tributaire d'un empirisme ou un pragmatisme irraisonné comme celui que dépeint Jacques Léonard? On ne saurait l'affirmer à partir de cette source. Cependant, la centration sur le patient, le réajustement constant du traitement administré, le recours à de multiples substances, bref, le déploiement d'un jeu complexe de dosages et de combinaison s'avèrent des caractéristiques indéniables de «l'art de prescrire » exercé par les médecins. Et ce, à une époque où le discours médical reconnaît d'emblée que la pharmacopée n'est pas encore des plus efficaces.

Il faut voir, en fait, qu'à la même époque, un tout autre type de recours à la médication, essentiellement populaire celui-ci, se déploie avec force. En effet, au moment où les médecins construisent dans

46. UMC, $13(1884): 386$. 
leurs pratiques et justifient à travers leur discours un rapport à la thérapeutique qu'ils veulent fondé sur des savoirs et sur un raisonnement scientifique, l'industrie du remède secret est en plein essor ${ }^{47}$. Le remède secret, pourtant, constitue en quelque sorte l'antithèse de la prescription médicale. Il est aussi simple qu'elle est complexe; aussi universel qu'elle est spécifique. Se peut-il dès lors que l'accentuation de ce mode d'usage de la matière médicale constitue symboliquement un moyen d'affirmation professionnelle de la part des médecins face à la concurrence de plus en plus forte de l'industrie du remède secret?

\section{LE RECOURS À LA THÉRAPEUTIQUE COMME ASSISE D'UNE IDENTITÉ PROFESSIONNELLE?}

Dans l'interprétation des transformations de la pratique médicale au $\mathrm{XIX}^{\mathrm{e}}$ siècle, les travaux de John Warner ont mis en évidence la dimension symbolique que revêt l'usage de la thérapeutique ${ }^{48}$. Aux États-Unis, la profession médicale ne jouit d'aucune reconnaissance légale avant la fin du siècle et les médecins subissent très fortement la concurrence des sectes ${ }^{49}$. Dans un tel contexte, Warner a montré comment un certain usage de la thérapeutique avait constitué pour les médecins américains une façon de se distinguer face aux pratiques parallèles, devenant du même souffle un élément clé dans la construction de l'image identitaire de la profession. L'abandon relativement tardif de la médecine héroïque par les praticiens américains et sa réapparition temporaire comme réaction face à l'accentuation de la concurrence s'expliquerait en partie par l'importance symbolique que revêt l'usage de la thérapeutique comme moyen d'affirmation professionnelle.

Au Québec, les pratiques parallèles à la médecine orthodoxe apparaissent peu nombreuses et peu développées, bien qu'il existe, à compter de 1865 , un Collège d'homéopathie ${ }^{50}$. De plus, contrairement à la situa-

47. C'est en effet à partir des années 1860 que l'on constate à travers la presse populaire l'omniprésence de la publicité concernant ces remèdes. Le changement s'opère assez radicalement au cours des années 1850-1860. À la fin du siècle, plusieurs milliers de ces remèdes auront envahi le marché québécois. Voir J. Collin et D. Béliveau, op. cit.

48. J. H. Warner, «Power, Conflict, and Identity in Mid-Nineteenth-Century American Medicine : Therapeutic Change at the Commercial Hospital in Cincinnati », Journal of American History, 73 (1987) : 934-956.

49. R. L. Numbers, « Do it Yourself the Sectarian Way », dans W. Laevett et R. L. Numbers, dir., Sickness and Health in America (Madison, University of Wisconsin Press, 1978).

50. Jean-Pierre Robitaille, «L'homéopathie au Québec (1840-1904) : l'institutionnalisation d'une pratique médicale controversée », Revue d'histoire de l'Amérique française, 50,3 (hiver 1997) : 347-374. 
tion qui prévaut aux États-Unis, les médecins dominent assez nettement le champ de la santé. Par contre, l'industrie du remède secret, alors en plein essor, échappe largement à leur contrôle. C'est de ce côté, semblet-il, qu'émane la plus redoutable concurrence pour les médecins ${ }^{51}$.

Dès la seconde moitié du XIX ${ }^{\mathrm{e}}$ siècle, les diverses préparations (sirops, toniques, purgatifs) prescrites par les médecins et composées par les pharmaciens sont progressivement concurrencées par celles que fabriquent, à grande échelle, une industrie des remèdes secrets en plein essor. Destinés, le plus souvent, au traitement des maux chroniques, durables ou douloureux, ces médicaments brevetés s'immiscent peu à peu dans le quotidien des populations. Les « maladies de poitrine » (allant de la simple toux jusqu'à la phtisie) ou du système digestif (dyspepsie, dysenterie, etc.) de même que les affections cutanées issues du mal vénérien sont fréquemment visées par ces remèdes aux innombrables vertus. Au dire de leurs promoteurs toutefois, la portée thérapeutique des médicaments brevetés ne s'arrête pas là. Ils parviennent même à guérir le « mal-être » épuisement, langueur, douleurs incessantes.

Or ces remèdes sont facilement accessibles. Ne requérant pas d'ordonnance, ils peuvent être vendus par les épiciers, les marchands généraux tout aussi bien que par les pharmaciens. Ils ne sont pas pour autant inoffensifs. Certains renferment de puissants narcotiques ou de fortes concentrations d'alcool. La publicité qui en est faite préconise, néanmoins, une consommation régulière, voire quotidienne de ces produits. Aux yeux d'une large fraction de la population aux prises avec les problèmes que suscitent l'industrialisation et l'urbanisation anarchique qui l'accompagne, les remèdes secrets incarnent vraisemblablement ce qu'il y a de plus efficace contre la maladie.

On évalue mal la quantité exacte de ces produits sur le marché. Ils sont toutefois omniprésents à travers la publicité dans les journaux à partir des années 1870. En 1899, certains experts avanceront d'ailleurs que leur nombre s'élève à plusieurs milliers sur le marché québécois. À la production nationale se mêle en effet celle qui nous vient alors de l'étranger : plus de 1000 produits différents en provenance de nos voisins du Sud, près de 400 en provenance de l'Europe,

51. À ce sujet, pour l'ensemble du Canada, voir Daniel J. Malleck, Refining Poison, Defining Power : Medical Authority and the Creation of Canadian Drug Prohibition Laws, 1800-1908, thèse de doctorat, Queen's University, 1998. 
selon le catalogue de la Lyman, Sons \& Co., le plus important grossiste montréalais et canadien en produits pharmaceutiques ${ }^{52}$.

La publicité, d'ailleurs, témoigne de l'engouement pour ces produits en même temps qu'elle le suscite. Dès les années 1860 , les annonces de remèdes secrets occupent une part importante de l'espace publicitaire de la presse populaire ${ }^{53}$. L'incitation à l'automédication constitue l'élément central du discours publicitaire concernant les médicaments brevetés. Il s'agit bien, en effet, de suggérer un usage régulier des remèdes qui sont vantés ${ }^{54}$.

L'usage répandu de produits susceptibles de contenir d'importantes doses de stupéfiants ou d'alcool ne concerne d'ailleurs pas uniquement les populations adultes, mais touche également les enfants. Une variété impressionnante de sirops calmants à base d'opium ou de morphine leur sont destinés. Or la publicité qui incite à leur utilisation s'avère d'autant plus insidieuse qu'elle attise le doute et l'inquiétude des mères par rapport aux maladies d'enfants et ce, à une époque où le taux de mortalité infantile est extrêmement élevé. Dans une réclame se rapportant au German Syrop, on peut lire que « [...] les maladies des enfants viennent vite et finissent d'une manière fatale ou autrement en très peu de temps ${ }^{55} »$. Dans tous les cas, il faut agir rapidement. Attendre l'avis du médecin, avance-t-on, peut être fatal. Nul besoin, du reste, d'un diagnostic précis puisque ces sirops pour enfants embrassent un vaste éventail de maladies possibles.

Au tournant du siècle, à l'occasion d'une enquête visant à déterminer si les remèdes secrets peuvent être vendus en épicerie ou s'ils doivent ne demeurer accessibles qu'en pharmacie, les médecins interrogés dénonceront ainsi les effets pervers de l'automédication. Ils déploreront l'absence de contrôle qui « encourage les gens vraiment malades à se servir de ces remèdes et les empêche de consulter un médecin jusqu'au moment où la maladie a fait de tels progrès, qu'il

52. Ces informations proviennent du catalogue des Patent Medicines and Proprietary Preparations disponibles chez le grossiste Lyman, Sons \& Co. en 1896, qui constitue l'une des plus importantes entreprises pharmaceutiques canadiennes à cette époque. Pour plus de détails, voir J. Collin et D. Béliveau, Histoire de la pharmacie..., chapitre 4.

53. Nous avons procédé à un examen attentif de quelques journaux populaires de Montréal et de villes plus modestes : La Gazette de Trois-Rivières (entre 1817 et 1821); le Montreal Herald (1822-1826); La Minerve (1828-1832 et 1870); Le Journal des Trois-Rivières (1866-1870 et 18801894) et Le Courrier de Saint-Hyacinthe (1866-1870 et 1890-1894).

54. C'est dès lors en ces termes que le promoteur du Female Porous Plaster et du Régulateur de la Santé des femmes s'adresse à ses clientes : «Une cuillérée à soupe du "Régulateur" pris avant chaque repas relève les forces, arrête toute douleur, donne appétit et facilite la digestion. » Ce « tonique des nerfs par excellence », qui « dispose à la gaité et chasse les voiles sombres de la mélancolie » requiert effectivement une utilisation constante. Le Courrier de Saint-Hyacinthe, 18 août 1891, 3.

55. Le Courrier de Saint-Hyacinthe, 20 octobre 1891, 2. 
est souvent trop tard pour y remédier $[\ldots]^{56} \gg$. En revanche, les épiciers et les fabricants insisteront, quant à eux, sur la similitude entre les remèdes à formule secrète et les ordonnances prescrites par les médecins. Selon un fabricant, « [...] les médecines brevetées ne sont ni plus ni moins qu'une prescription bien faite [...]». Ce dernier admet, certes, que ces remèdes peuvent être dangereux lorsque le malade ne respecte pas la posologie indiquée par le fabricant; toutefois, il s'empresse d'ajouter qu'il est très difficile d'intervenir dans les décisions prises par le consommateur, à partir de conseils, d'avis divers ou de publicité ${ }^{57}$. Les fabricants et épiciers proclament également la capacité des consommateurs à décider eux-mêmes de leur médication. L'intérêt public, fait-on valoir, commande que ces remèdes soient totalement accessibles, puisque les plus démunis sont les acheteurs les plus assidus de ces produits : « [...] en permettant la vente par l'épicier, ça permet au pauvre de se le procurer plus facilement. Pour le pauvre, pour ceux qui n'ont pas le moyen de payer le médecin, c'est toujours un soulagement et une facilité qui leur sont offerts ${ }^{58}$. »

Si la concurrence des remèdes secrets se perçoit à travers les témoignages, elle se fonde en partie sur les similitudes des produits qui sont offerts aux patients. Les préparations officinales comptent en effet pour près de la moitié des ingrédients entrant dans la composition des médications prescrites par les médecins (tableau 2). Pour l'essentiel, il s'agit de sirops, d'élixirs, de teintures, de poudres et de vins. Or les remèdes secrets se présentent également sous la forme de vins, sirops et liqueurs et constituent, en quelque sorte, un produit simplifié et souvent altéré par rapport à la recette inscrite dans la pharmacopée puisqu'on y aura ajouté plus d'alcool ou encore d'opium que ce qui est recommandé.

Toutefois, en dépit d'apparences et de formes similaires, remèdes secrets et prescriptions médicales divergent quant à la philosophie qui les sous-tend. Les remèdes secrets sont fondés sur le principe de l'universalité : ils soignent une multitude de symptômes disparates et ce, indépendamment de l'environ-

56. Voir Comité permanent de Législation et des lois expirantes, enquête tenue devant le Comité de Législation sur le bill intitulé : «Loi amendant la Loi de pharmacie de Québec », Journal de l'Assemblée législative de la province de Québec, du 12 janvier au 10 mars 1899, Appendice B, 423.

57. Et il poursuit : «Dans la généralité des cas, l'acheteur vient acheter à la pharmacie ou chez l'épicier cette médecine soit sur le conseil d'un parent ou d'un ami ou d'une annonce dans les journaux. J'ai constaté moi-même que les gens qui viennent pour acheter une bouteille de médecine brevetée viennent avec l'intention de l'avoir et nulle autre que celle-là. Si on veut les détourner, ils ne veulent pas en prendre une autre. » Comité permanent de Législation et des lois expirantes, «Loi amendant la Loi de pharmacie de Québec », op. cit., 436.

58. Ibid., 437. 
nement et de l'histoire médicale des consommateurs. Ils se fondent également sur le principe de la standardisation, notamment à travers le processus de fabrication industrielle, mais également parce que la publicité qui s'y rattache suggère la consommation d'un remède unique jusqu'à ce que la maladie, quelle qu'elle soit, disparaisse. À l'inverse, la démarche du médecin s'appuie, quant à elle, sur le principe de la spécificité du patient et de son environnement ainsi que sur la non-standardisation de la médication prescrite. Selon cette logique, il est nécessaire de réévaluer sans cesse l'évolution de la maladie et la composition des médicaments destinés à la contrer. C'est donc sur le plan de la philosophie, de l'approche ainsi que sur celui de la valeur symbolique accordée à l'acte de prescrire que se situent les différences profondes.

L'acte de prescrire, qui se caractérise par la variété de substances utilisées et par l'absence de répétitions dans les stratégies thérapeutiques, est totalement antinomique par rapport à ce que prône l'industrie pharmaceutique. Dès lors, l'attachement des médecins à cette démarche est peut-être d'autant plus fort que celle-ci incarne le symbole d'un savoir poussé, inaccessible au profane et seul capable de permettre un usage efficace et spécifique de l'arsenal thérapeutique. La prescription constitue alors le signe tangible d'une expertise irremplaçable en même temps que la manifestation du dévouement et de l'intérêt que porte le médecin à ses patients.

$$
* \quad * \quad *
$$

Cette analyse du discours et des pratiques médicales face à l'arsenal thérapeutique dans le dernier tiers du XIX ${ }^{\mathrm{e}}$ siècle démontre qu'il n'y a plus trace, à cette époque, du scepticisme suggéré a posteriori par certains auteurs. Toutefois, la valorisation d'un « art de prescrire » fondé sur un rapport complexe et polymorphe à l'arsenal thérapeutique ne saurait pour autant s'expliquer par les seuls progrès scientifiques qui marquent la période. Les médecins qui s'expriment à travers la presse médicale reconnaissent d'emblée les limites des moyens thérapeutiques qui sont à leur disposition et ce, malgré l'accroissement considérable de la pharmacopée. C'est dire que d'autres dynamiques sont également à l'œuvre pour expliquer leur recours à l'arsenal thérapeutique et la place qu'occupe alors le médicament dans leur pratique médicale. Dans le contexte de concurrence que nous avons dépeint, l' « art de prescrire » a pu constituer un moyen d'affirmation professionnelle. Toutefois, seule une analyse approfondie de l'ordonnancier jusqu' au début du $\mathrm{XX}^{\mathrm{e}}$ siècle nous permettra de confirmer ou d'étayer cette hypothèse et surtout de déceler le changement de paradigme qui préfigure l'entrée dans le $\mathrm{XX}^{\mathrm{e}}$ siècle, la simplification des pratiques de prescription vers l'ingrédient unique et le report des symboles de l'expertise médicale vers d'autres objets. 
ANNEXE 1

\section{Liste des substances maîtresses inscrites à l'ordonnancier 1869-1890}

\author{
Absynthe officinale \\ Acétanilide \\ Acide acétique \\ Acide arsénieux \\ Acide benzoïque \\ Acide borique \\ Acide bromhydrique \\ Acide chlorhydrique \\ Acide chloro-azotique \\ Acide chromique \\ Acide chrysophanique \\ Acide citrique \\ Acide cyanhydrique \\ Acide gallique \\ Acide lactique \\ Acide nitrique \\ Acide oxalique \\ Acide phosphorique \\ Acide salicylique \\ Acide sulfureux \\ Acide sulfurique \\ Acide tannique \\ Acide tartrique \\ Aconite \\ Aconitine \\ Actée à grappes \\ Agaric blanc officinal \\ Alcool \\ Alétris farineux \\ Aloès \\ Aloin \\ Aluminium \\ Alun \\ Amande \\ Amande amère \\ Amande douce \\ Amidon \\ Ammoniaque \\ Ammonium \\ Anémone pulsatille \\ Aneth \\ Anis
}
Antimoine
Antipyrine
Apiol
Argent
Aristol
Arnica
Ase-fétide
Atropine
Badiane
Balsamite
Baume de tolu
Baume du Pérou
Belladone
Belle-angélique
Benjoin
Bergamote
Bismuth
Bœuf
Bois de campêche
Borax
Brome
Buchu
Cacao
Cachou
Cade
Caféine
Cajeput
Calabar
Calamine
Calcium
Camomille
Camphre
Cannelle
Cantharides
Caramel
Cardamome
Carvi
Cascara segrada
Castor
Castoreum
Cèdre de Virginie
Cenelle 
Cerf

Cerisier noir

Cérium

Chanvre indien

Chapelure

Charbon végétal

Chaulmoogra

Chaux

Chêne

Chicorée

Chiendent officinal

Chiretta

Chloral

Chloralamide

Chlore

Chloroforme

Chrysarobine

Ciguë

Cinchonidine

Cire

Citron

Citrouille

Coca

Cocaïne

Cochenille

Codéine

Coing

Colchicine

Colchique

Collodion

Colombo

Coloquinte

Copahu

Coriandre

Coton

Craie

Créoline

Créosote

Croton Tiglii

Cubèbe

Cuivre

Cynoglosse

Digitale

Digitaline

Duboisine

Eau

Élatérine

Élatérium

Ergot de seigle
Erigeron du Canada

Ésérine

Éther

Eucalyptol

Eucalyptus

Fenouil

Fenugrec

Fer

Fougère mâle

Framboise

Fusain

Gaïac

Galbanum

Gamboge

Gelsemium

Genêts

Genièvre

Gentiane

Gingembre

Girofle

Glycérine

Gomme adragante

Gomme arabique

Gomme shellac

Goudron

Graisse de baleine

Graisse de porc

Grenadier

Grindelia robusta

Guarana

Guimauve

Gutta percha

Hamamélis Virginica

Hellebore verte

Houblon

Hydrastis

Indéfinie

Iode

Iodoforme

Iodol

Ipécacuanha

Iris

Jaborandi

Jalap

Jalapine

Jambol

Jargonnelle pair

Jusquiame

Jute 
Kamala

Kino

Lactopeptine

Lactose

Lactucarium

Lanoléine

Laurier-cerise

Lavande

Leptandrin

Lin

Lippia Mexicana

Lithium

Lobélie enflée

Lupin

Lupuline

Lycopode

Magnésium

Maiis

Malt

Manganèse

Manne

Matico

Mauve

Menthe poivrée

Menthe verte

Menthol

Ményanthe

Mercure

Méthylacétanilide

Miel

Morphine

Morue

Moutarde

Muguet

Mûrier des haies

Musc

Myrrhe

Naphtaline

Naphtol

Nerprun pourpre

Nitroglycérine

Noix de galle

Noix de muscade

Noix vomique

Euf

Olive

Opium

Or

Orange
Orange amère

Orge

Origan

Orme

Palommier

Pancréatine

Paraffine

Pareira brava

Pavot blanc

Pepsine

Persicaire poivrée

Phénacétine

Phénol

Phosphore

Pichi

Pilocarpine

Piment

Pin du Canada

Pissenlit

Plomb

Podophylle

Poivre de Guinée

Poivre noir

Poix blanche

Polygale de Virginie

Potassium

Prune

Pyrèthre

Pyrogallol

Pyrole

Quassia

Quassine

Quebracho

Quinine

Quinquina jaune

Quinquina rouge

Raifort

Raisins d'ours

Ratanhia

Réglisse

Résine

Résorcine

Rhubarbe

Romarin

Ronce

Rose

Rose à centfeuilles

Rose de Damas

Rue 
Sabine

Safran

Salicine

Salol

Salsepareille

Sang-dragon

Santal citrin

Santonine

Saule

Savon

Scammonée

Scille

Seigle

Sel

Sené

Serpentaire de Virginie

Sésame

Sodium

Soufre

Spartéine

Spigélie

Storax

Stramoine
Strophantus

Strychnine

Succin

Sucre

Sulfonal

Sumac

Sumbul

Sureau

Tamarind

Térébenthine

Térébenthine du Canada

Thapsia

Thymol

Tilleuil

Valériane

Vanille

Vaseline

Vernis du Japon

Verveine commune

Viburnum

Vin

Yerba santa

Zinc 